\title{
Dynamics and Fate of Beneficial Mutations Under Lineage Contamination by Linked Deleterious Mutations
}

\author{
Sophie Pénisson, * Tanya Singh, ${ }^{+}$Paul Sniegowski, ${ }^{\dagger}$ and Philip Gerrish ${ }^{\dagger, \S, * *}$ \\ *Université Paris-Est, Laboratoire d'Analyse et de Mathématiques Appliquées (UMR 8050), UPEMLV, UPEC, CNRS, F-94010, Créteil, \\ France, ${ }^{\dagger}$ Department of Biology, Leidy Laboratories, University of Pennsylvania, Philadelphia, Pennsylvania 19104, ₹School of \\ Biological Sciences, Georgia Institute of Technology, Atlanta, Georgia 30332, §Theoretical Biology and Biophysics, Theoretical \\ Division, Los Alamos National Laboratory, New Mexico 87545, and **Departamento de Ciencias Químico-Biológicas, Instituto de \\ Ciencias Biomedicas, Universidad Autónoma de Ciudad Juárez, Chihuahua, México, C.P. 32310
}

\begin{abstract}
Beneficial mutations drive adaptive evolution, yet their selective advantage does not ensure their fixation. Haldane's application of single-type branching process theory showed that genetic drift alone could cause the extinction of newly arising beneficial mutations with high probability. With linkage, deleterious mutations will affect the dynamics of beneficial mutations and might further increase their extinction probability. Here, we model the lineage dynamics of a newly arising beneficial mutation as a multitype branching process. Our approach accounts for the combined effects of drift and the stochastic accumulation of linked deleterious mutations, which we call lineage contamination. We first study the lineage-contamination phenomenon in isolation, deriving dynamics and survival probabilities (the complement of extinction probabilities) of beneficial lineages. We find that survival probability is zero when $U \geq s_{b}$, where $U$ is deleterious mutation rate and $s_{b}$ is the selective advantage of the beneficial mutation in question, and is otherwise depressed below classical predictions by a factor bounded from below by $\sim 1-U / s_{b}$. We then put the lineage contamination phenomenon into the context of an evolving population by incorporating the effects of background selection. We find that, under the combined effects of lineage contamination and background selection, ensemble survival probability is never zero but is depressed below classical predictions by a factor bounded from below by $e^{-\varepsilon U / \bar{s}_{b}}$, where $\bar{s}_{\mathrm{b}}$ is mean selective advantage of beneficial mutations, and $\varepsilon=1-e^{-1} \approx 0.63$. This factor, and other bounds derived from it, are independent of the fitness effects of deleterious mutations. At high enough mutation rates, lineage contamination can depress fixation probabilities to values that approach zero. This fact suggests that high mutation rates can, perhaps paradoxically, (1) alleviate competition among beneficial mutations, or (2) potentially even shut down the adaptive process. We derive critical mutation rates above which these two events become likely.
\end{abstract}

KEYWORDS adaptation; mutation rates; fixation; multitype branching processes

ENEFICIAL mutations are the ultimate source of the genetic variation that fuels evolutionary adaptation, but deleterious mutations are likely to be far more abundant (Sturtevant 1937; Muller 1950). Perhaps for the sake of simplicity, the evolutionary effects of these two types of fitnessaffecting mutations were generally considered separately in early studies. For example, Muller (1964) assumed that ben-

Copyright @ 2017 by the Genetics Society of America

doi: 10.1534/genetics.116.194597

Manuscript received August 8, 2016; accepted for publication January 4, 2017 . published Early Online January 18, 2017.

Supplemental material is available online at www.genetics.org/lookup/suppl/doi:10. 1534/genetics.116.194597/-/DC1.

${ }^{1}$ Corresponding author: School of Biological Sciences, Georgia Institute of Technology, 310 Ferst Dr., Atlanta, GA 30332. E-mail: pgerrish@gatech.edu eficial mutations were negligible and reasoned verbally that deleterious mutations should have disastrous consequences for populations in the absence of recombination because of the recurrent, stochastic loss of genotypic classes with the fewest deleterious mutations-Muller's ratchet (Felsenstein 1974). Haldane (1927), on the other hand, focused on the fate of single beneficial mutations in the absence of other fitness-affecting mutations and used single-type branching process theory to show that most such beneficial mutations are lost to what is now called genetic drift: the fixation probability of such a beneficial mutation is only about twice its selective effect, $s_{\mathrm{b}}$, for small $s_{\mathrm{b}}$.

In reality, of course, multiple fitness-affecting mutations (both beneficial and deleterious) can be present simultaneously 
in populations, and these mutations can influence each others' fates and evolutionary effects as a consequence of linkage (reviewed in Gordo and Charlesworth 2001; Barton 2009; Charlesworth 2009, 2013). Interactions between beneficial and deleterious mutations are of particular interest in this regard, because such interactions - in contrast to interactions between beneficial mutations alone - can determine whether a population will increase or decrease in fitness. Indeed, recent studies (Poon and Otto 2000; Bachtrog and Gordo 2004; Silander et al. 2007; Kaiser and Charlesworth 2009; Goyal et al. 2012) have indicated that beneficial mutations (including reversions of deleterious mutations) can impede or halt the fitness loss predicted in asexual populations under Muller's ratchet, as originally suggested by Haigh (1978). Moreover, a number of theoretical studies (Peck 1994; Barton 1995; Johnson and Barton 2002; Bachtrog and Gordo 2004; Jiang et al. 2011; Charlesworth 2013; McFarland et al. 2014) have shown that Haldane's classical fixation probability of $2 s_{\mathrm{b}}$ for a beneficial mutation can be reduced by the effects of selection against linked deleterious mutations (Birky and Walsh 1988; Campos 2004; Campos and Wahl 2010; Hartfield et al. 2010; Good and Desai 2014): in principle, such effects include both background selection against deleterious mutations already present in the genome on which the beneficial mutation appears, and selection against deleterious mutations that arise and accumulate in genomes carrying the beneficial mutation. The latter form of selective effect has not previously been singled out and analyzed as a population process in its own right; it is the primary focus of the current article and will be referred to as lineage contamination.

In preliminary computer simulations, we observed that the fixation probability of a beneficial mutation appearing in an otherwise initially homogeneous asexual population with a high genomic mutation rate is considerably reduced below Haldane's classical $2 s_{\mathrm{b}}$ expectation. Because the population was initially homogeneous in fitness, the reduction below classical predictions could not be attributed to background selection. We hypothesized that the lower probability of fixation of a beneficial mutation in this situation can be attributed to lineage contamination: specifically, Muller's ratchet operates at a much faster rate in the small lineage founded by the beneficial mutation than in the rest of the population. Here, we present the results of analytical modeling and further computer simulations that support this hypothesis, elucidate the mechanics of lineage contamination in detail, and show how lineage contamination affects fixation probabilities and derivative quantities.

We model the influences of background selection and lineage contamination, both singly and jointly, on the fate of beneficial mutations. Under background selection alone, a beneficial mutation that lands on the best genetic background (the one least loaded with deleterious mutations) always has a nonzero probability of achieving fixation in an asexual population. In contrast, under lineage contamination alone, a beneficial mutation can have a probability of survival that is zero if the mutation rate is high enough. Our simulations and analytical results suggest that when both background selection and lineage contamination are operating - as they do in real populations-asexual populations traverse a continuum of evolutionary regimes as the genomic mutation rate increases. At low mutation rates, beneficial mutations appear infrequently enough that they do not interfere with each others' progress to fixation (the "periodic-selection" regime; Sniegowski and Gerrish 2010); as the mutation rate increases, alternative beneficial mutations begin to compete with each other (the "clonal-interference" regime); as mutation rate increases further, we find that lineage contamination can suppress a fraction of beneficial mutations that is sufficient to cause a population to revert to the periodic selection regime; and ultimately, at very high mutation rates, a regime can be reached in which beneficial mutations are no longer substituting. Significantly, these last two regimes would not obtain without the operation of lineage contamination: at high mutation rates, background selection alone cannot alleviate clonal interference or significantly impede the adaptive substitution process, but lineage contamination can. Our results, therefore, indicate that the lineage contamination effect is central to determining the adaptive fate of a population when both beneficial and deleterious mutations arise (Bull et al. 2007; Bull and Wilke 2008; Springman et al. 2009).

Our models assume: (1) that the environment remains constant over the relevant time span, (2) there is complete linkage between the beneficial and deleterious mutations in question, (3) fitness effects of mutations are multiplicative (no epistasis), and (4) there are no frequency-dependent effects other that the one studied here (i.e., the one due to differential rates of Muller's ratchet). While our branching process model assumes replication by binary fission, we show that relaxing this assumption only alters our results by a constant factor. To show this, we give an alternative derivation of survival probability bounds that implements the model of Johnson and Barton (2002); this model assumes that numbers of offspring follow a Poisson distribution, and it simply changes survival probabilities by a factor of two.

It will facilitate further reading to precisely define three terms: extinction probability, $p_{\text {ext }}$, is the probability that a beneficial lineage, arising in an otherwise infinite population, becomes extinct in finite time; survival probability is the complement of the extinction probability $p_{\text {svl }}=1-p_{\text {ext }}$; and finally, fixation probability, $p_{\mathrm{fix}}$, is the probability that a lineage will displace the rest of a finite population (become fixed) in finite time. We further note that it is possible for an ultimately doomed lineage to become fixed in a finite population, implying $p_{\mathrm{fix}}>p_{\mathrm{svl}}$.

\section{Theory 1: Lineage contamination in isolation}

In this section, we model the random accumulation of deleterious mutations in a growing lineage founded by the occurrence of a single beneficial mutation (henceforth, beneficial lineage) of known selective advantage $s_{\mathrm{b}}$ within an otherwise homogeneous population. 


\section{Multitype branching process model}

Our stochastic model is a discrete-time multitype branching process, where a "type" $i \in \mathbb{N}$ corresponds to the number of acquired deleterious mutations. The model describes the evolution of the composition of the population $\mathbf{X}_{t}=\left(X_{t, 0}, X_{t, 1}, \ldots\right)$, $X_{t, i}$ being the number of individuals carrying $i$ deleterious mutations at time $t \in \mathbb{N}$. We denote by $U \geq 0$ the deleterious mutation rate, and by $0 \leq s_{\mathrm{b}} \leq 1$ and $0<s_{\mathrm{d}}<1$ the selective advantage of beneficial mutations and disadvantage of deleterious mutations, respectively. The fitness of an individual carrying $i$ deleterious mutations is:

$$
w_{i}=\left(1+s_{\mathrm{b}}\right)\left(1-s_{\mathrm{d}}\right)^{i} .
$$

The model can be described as follows: at each time-step, each individual produces two descendants carrying as many deleterious mutations as itself. Each descendant might accumulate during this reproduction $k$ additional deleterious mutations, with probability $e^{-U} U^{k} / k$ !. If the parent was of type $i$, the descendant is then of type $i+k$ and is selected according to its fitness, i.e., with probability proportional to $w_{i+k}$. Therefore, an individual of type $i$ produces a total number of 0,1 , or 2 descendants, each of them being of a type greater than or equal to $i$. We refer to the Supplemental Material, File S1, for a more detailed description of the model.

We consider a subpopulation carrying a beneficial mutation (i.e., a single beneficial lineage) arising in a large wildtype population. To study the lineage-contamination effect in isolation, we assume that both populations initially do not carry any deleterious mutations. For this purpose we consider two independent branching processes: $\left(\mathbf{X}_{t}\right)_{t \in \mathbb{N}}$, describing the evolution of a wild-type population of initial size $N$, hence with $s_{\mathrm{b}}=0$ and initial state $\mathbf{X}_{0}=(N, 0,0, \ldots)$; and $\left(\mathbf{X}_{t}^{b}\right)_{t \in \mathbb{N}}$, describing the evolution of a single beneficial lineage, with $s_{\mathrm{b}}>0$ and initial state $\mathbf{X}_{0}^{b}=(1,0,0, \ldots)$.

\section{Mean demographic dynamics of each subpopulation}

The mean wild-type population size (all types combined) at time $t$ is given by $N e^{-U t} e^{U\left[1-\left(1-s_{\mathrm{d}}\right)^{t}\right]\left(1-s_{\mathrm{d}}\right) / s_{\mathrm{d}}}$ (Supplemental Equation S4, File S1). A beneficial mutation occurring within the wild-type population founds a beneficial lineage whose mean size is given by $\left(1+s_{\mathrm{b}}\right)^{t} e^{-U t} e^{U\left[1-\left(1-s_{\mathrm{d}}\right)^{t}\right]\left(1-s_{\mathrm{d}}\right) / s_{\mathrm{d}}}$ (S5). Note that as time tends to infinity this quantity tends to $+\infty$ if $U<\ln \left(1+s_{\mathrm{b}}\right)$, to $e^{U\left(1-s_{\mathrm{d}}\right) / s_{\mathrm{d}}}$ if $U=\ln \left(1+s_{\mathrm{b}}\right)$, and to 0 if $U>\ln \left(1+s_{\mathrm{b}}\right)$. The latter convergence will typically not be monotonic (Figure S1 in File S1).

\section{Extinction and survival probabilities}

The previous result concerning the evolution of the mean beneficial lineage population size can be refined by looking at the extinction probability of the beneficial lineage. By this we mean the probability $p_{\text {ext }}$ that the process $\left(\mathbf{X}_{t}^{b}\right)_{t \in \mathrm{N}}$ does become extinct, i.e., $p_{\text {ext }}=\mathbb{P}\left(\forall i \in \mathbb{N}, \lim _{t \rightarrow \infty} X_{t, i}^{b}=0\right)$. We show (Proposition 1; see File S1) that although the number of types is infinite, this probability also equals $\mathbb{P}\left(\lim _{t \rightarrow \infty} \sum_{i \in \mathbb{N}} X_{t, i}^{b}=0\right)$. The beneficial lineage almost surely becomes extinct if and only if $U \geq U_{c}$, where the critical deleterious mutation rate is given by

$$
U_{c}=\ln \left(1+s_{\mathrm{b}}\right) .
$$

Of course, this implies that if $U<U_{c}$ then $p_{\text {ext }}<1$, i.e., the beneficial lineage can survive with positive probability.

\section{Bounds}

The number of equations required to determine this probability, $D_{0}+1$, is finite and can be small: $D_{0}=\sup \left\{i \in \mathbb{N}: e^{-U} w_{i}>1\right\}$ Thus only one equation is required $\left(D_{0}=0\right)$ whenever $s_{\mathrm{d}} \geq 1-e^{U}\left(1+s_{\mathrm{b}}\right)^{-1}$. We find (File S1) that survival probability $p_{\mathrm{svl}}=1-p_{\mathrm{ext}}$ is bounded by:

$$
p_{\text {svl }}= \begin{cases}K \tilde{s}_{\mathrm{b}} C, & U<U_{c}, s_{\mathrm{d}} \geq 1-e^{U}\left(1+s_{\mathrm{b}}\right)^{-1} \\ K \tilde{s}_{\mathrm{b}}, & U<U_{c}, s_{\mathrm{d}} \rightarrow 0 \\ 0, & U \geq U_{c},\end{cases}
$$

where $C=e^{U}\left[1-\left(e^{U}-1\right) / s_{\mathrm{b}}\right] \approx 1-U / s_{\mathrm{b}}$, and $\tilde{s}_{\mathrm{b}}=s_{\mathrm{b}}(1+$ $\left.s_{\mathrm{b}}\right)^{-2}$. In what follows, we will refer to $K \tilde{s}_{\mathrm{b}}$ as the "classical" survival probability (i.e., nonextinction probability). This classical survival probability is rederived in File S1, where it is shown to be exact for binary fission $(K=4)$ and a good approximation (a slight improvement on Haldane's $2 s_{\mathrm{b}}$ ) for Poisson-distributed offspring $(K=2)$. Survival probabilities modified to account for lineage contamination in isolation are bounded by Equation 3. These bounds are derived in File S1 from our binary fission model $(K=4)$ and are exact. Also in File S1, these bounds are shown to derive directly from expansion of the Johnson and Barton (2002) formulation, which assumes Poisson-distributed offspring $(K=2)$.

Exact computation of $p_{\text {svl }}$ is achieved numerically using algorithm (S11) derived in File S1. In Figure S7 in File S1, we show how, when $U<U_{c}$, fixation probability as computed using (S11) decreases monotonically in $s_{\mathrm{d}}$ from the upper limit, achieved when $s_{\mathrm{d}} \rightarrow 0$, to the lower limit, achieved when $s_{\mathrm{d}} \geq 1-e^{U}\left(1+s_{\mathrm{b}}\right)^{-1}$. In addition, we provide a mathematical proof that $p_{\text {svl }}$ is monotonic in $s_{\mathrm{d}}$ (File $\mathrm{S} 1$ ); this gives rise to the fact that, when $U<\ln \left(1+s_{\mathrm{b}}\right) \approx s_{\mathrm{b}}$, survival probability is bounded as:

$$
K \tilde{s}_{\mathrm{b}} C \leq p_{\mathrm{svl}} \leq K \tilde{s}_{\mathrm{b}} .
$$

The fact that $p_{\text {sv }}$ is monotonic in $s_{\mathrm{d}}$ will also be key to the logic of our approximations (next section). Figure 1 plots exact computations of $p_{\text {svl }}$ (thin intermediate curves) as well as limiting cases given by Equation 3 (thick curves) as a function of the deleterious mutation rate $U$ and selective advantage $s_{\mathrm{b}}$, respectively.

\section{Fixation probabilities}

In this branching process model, the fixation probability of the beneficial lineage is the probability $p_{\text {fix }}$ that at some point the whole population carries the beneficial mutation. Note that because we take into account stochastic variation in population size, the beneficial mutation might not be permanently 



Figure 1 Survival probabilities as functions of $(A)$ deleterious mutation rate, and (B) selective advantage of the beneficial mutation. Plotted here are survival probabilities of a beneficial mutation in an otherwise homogeneous population (lineage contamination in isolation). Thick blue curves plot the case $s_{d}>s_{b}$, thick red curves plot the limit $s_{d} \rightarrow 0$, and thin curves of intermediate color plot survival probabilities for intermediate values of $s_{d}$ (computed by numerical solution of the survival probability equations given in File S1). Parameters are: $s_{b}=0.1$ for $(A)$, and $U=0.1$ for (B). The threshold phenomenon apparent in these plots may be understood as follows: when $U<\ln \left(1+s_{b}\right)$, the beneficial lineage is growing fast enough, relative to the mutation rate, that a mutation-free class (zero class) can be maintained; when $U>\ln \left(1+s_{b}\right)$, however, the zero class is lost and the lineage undergoes within-population mutational meltdown.

established even after fixation, because the population might eventually become extinct afterward. The fixation probability here corresponds exactly to the probability that the wildtype population dies out before the single beneficial lineage does: $p_{\text {fix }}=\mathbb{P}\left(T_{\text {ext }}<T_{\text {ext }}^{b}\right)$, where $T_{\text {ext }}=\inf \left\{t \in \mathbb{N}: \mathbf{X}_{t}=0\right\}$ (alternately, $T_{\text {ext }}^{b}$ ) is the extinction time of the wild-type population (alternatively, beneficial lineage). From what precedes, we know that $T_{\text {ext }}$ is almost surely finite, whereas $T_{\text {ext }}^{b}$ is almost surely finite if and only if $U \geq U_{c}$. Note also that the strict inclusion of the probability events $\left\{T_{\text {ext }}^{b}=+\infty\right\} \subsetneq\left\{T_{\text {ext }}<T_{\text {ext }}^{b}\right\}$ validates our previous statement that $1-p_{\text {ext }}<p_{\mathrm{fix}}$. This implies in particular that, in this model, the fixation probability is never zero. Although we cannot provide a closed-form expression for $p_{\mathrm{fix}}$, this

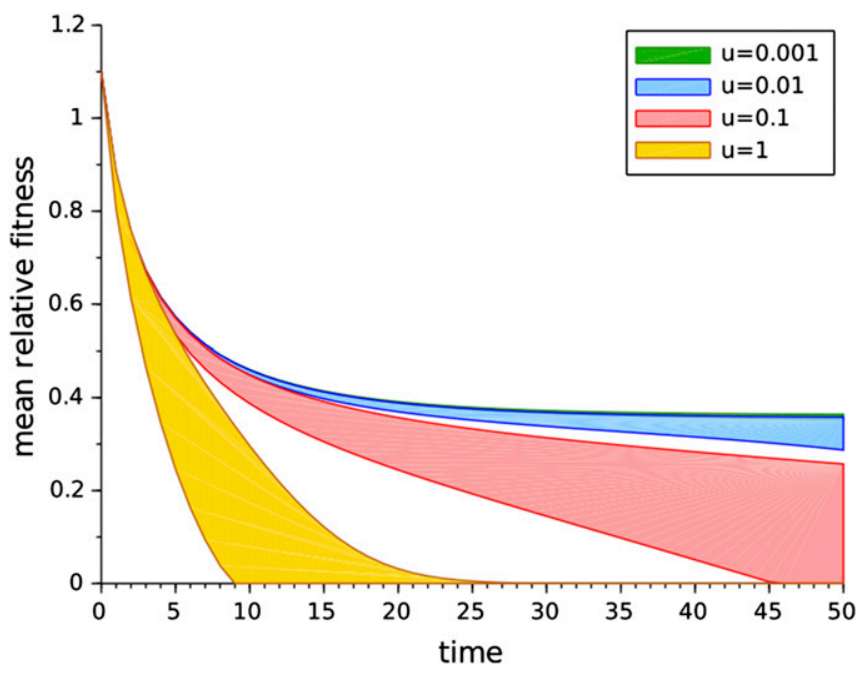

Figure 2 Evolution over time of the mean relative fitness of a single beneficial lineage $\left(s_{b}=0.1\right)$ in a large wild-type population, initially homogeneous, with $s_{d}=0.03$. Upper and lower bounds were obtained as described in File S1. The decrease in relative fitness is caused by the comparatively rapid accumulation of deleterious mutations owing to the small size of emerging beneficial lineages, i.e., lineage contamination.

probability can be well approximated numerically by (S14 and S15). We illustrate this result in Figure S5 in File S1, where we plot $p_{\text {fix }}$ as a function of the deleterious mutation rate.

\section{Fitness dynamics of a beneficial lineage within a population}

The fitness of the beneficial lineage at time $t$ is given by

$$
\mathcal{W}\left(\mathbf{X}_{t}^{b}\right)=\sum_{i \in \mathrm{N}} \frac{w_{i} X_{t, i}^{b}}{\sum_{i \in \mathrm{N}} X_{t, i}^{b}}
$$

Because of the potential extinction of the population, the random variable $\mathcal{W}\left(\mathbf{X}_{t}^{b}\right)$ is only defined for $t<T_{\text {ext }}^{b}$. We similarly define the fitness of the whole population $\mathcal{W}\left(\mathbf{X}_{t}+\mathbf{X}_{t}^{b}\right)$, and focus our study on the dynamics of the relative fitness $\mathcal{W}\left(\mathbf{X}_{t}^{b}\right) / \mathcal{W}\left(\mathbf{X}_{t}+\mathbf{X}_{t}^{b}\right)$. Because we assume in our model that the wild-type population is initially large, we approximate the relative fitness by its almost certain limit as $N$ tends to infinity (S16), namely

$$
e^{U\left(1-s_{\mathrm{d}}\right)\left[1-\left(1-s_{\mathrm{d}}\right)^{t}\right]} \mathcal{W}\left(\mathbf{X}_{t}^{b}\right)
$$

We also prove that the mean value of this relative fitness tends as time tends to infinity to $\left(1+s_{\mathrm{b}}\right) p_{\text {svl }}$ (S17 and S18). A plot of this long-term limit is given in Figure S6 in File S1. To have a more accurate description of the evolution of the relative fitness over time, we provide in addition an upper and lower bound (S19 and S20) of its mean value for each $t \in \mathbb{N}$, as illustrated in Figure 2.

\section{Mutational meltdown of a beneficial lineage}

Our goal here is to study the synergy between the loss of the least-loaded classes and the potentially decreasing size of the beneficial lineage. For technical reasons detailed in File S1, in 
this section we consider the continuous-time analog $\left(\mathbf{X}_{t}^{b}\right)_{t \geq 0}$ of the branching processes $\left(\mathbf{X}_{t}^{b}\right)_{t \in \mathrm{N}}$ studied previously. Assuming that at time $t$ the least-loaded class in the beneficial lineage's population is of type $i$, the process at this time is of the form $\mathbf{X}_{t}^{b}=\mathbf{n}_{i}=\left(0, \ldots, 0, n_{i i}, n_{i, i+1}, \ldots\right)$. Conditionally on $\mathbf{X}_{0}^{b}=\mathbf{n}_{i}$, we define the extinction time of the least-loaded class as $T_{i}=\inf \left\{t \geq 0: X_{t, i}^{b}=0\right\}$. The mutational-meltdown effect is then fully described by the sequence of random variables $T_{0}, T_{1}, \ldots$

Note that $T_{1}$ strongly depends on the random value $\mathbf{X}_{T_{0}}^{b}$ taken by the process at the beginning of the time interval $\left[T_{0}, T_{0}+T_{1}\right]$. Note also that assuming $\mathbf{X}_{T_{0}}^{b}=\mathbf{n}_{1}$, the strong Markov property enables study of the process on the latter interval to be reduced to its study on $\left[0, T_{1}\right]$, conditionally on $\mathbf{X}_{0}^{b}=\mathbf{n}_{1}$. We thus provide in Proposition 3 (File S1) an explicit computation of the cumulative distribution function $\mathbb{P}_{\mathbf{n}_{i}}\left(T_{i} \leq t\right)$ of the time to extinction of the least-loaded class of type $i$, for any $i$ and any initial condition $\mathbf{X}_{0}^{b}=\mathbf{n}_{i}$. From this we deduce its mean value $\mathbb{E}_{\mathbf{n}_{i}}\left(T_{i}\right)$. Again, three different regimes appear, depending on whether $U<U_{c}, U=U_{c}$, or $U>U_{c}$. We illustrate this result in Figure S2 and Figure S3 in File S1, where we plot the cumulative distribution function and mean value of the extinction time $T_{0}$ of the first leastloaded class, with $\mathbf{X}_{0}^{b}=\mathbf{n}_{0}=(1,0,0, \ldots)$.

Finally, to study not only the behavior of each extinction time separately but to take into account the stochastic evolution of the process $\left(\mathbf{X}_{t}^{b}\right)_{t \geq 0}$, we compute the sequence of the mean extinction times $\mathbb{E}_{\mathbf{n}_{0}}\left(T_{0}\right), \mathbb{E}_{\mathbf{n}_{1}}\left(T_{1}\right), \ldots$, where the deterministic sequence $\left(\mathbf{n}_{i}\right)_{i \in \mathrm{N}}$ is chosen to reflect as accurately as possible the mean evolution of $\left(\mathbf{X}_{t}^{b}\right)_{t \geq 0}$. We naturally choose $\mathbf{n}_{0}=(1,0,0, \ldots)$, and then define $\mathbf{n}_{1}$ as the mean value of the process at the end of the first time interval $\left[0, T_{0}\right]$. Because this mean value might not be integer valued, we round each of its coordinates to the closest integer. Hence we set $\mathbf{n}_{1}=\operatorname{round}\left[\mathbb{E}_{\mathbf{n}_{0}}\left(\mathbf{X}_{T_{0}}^{b}\right)\right]$, and iteratively define in a similar manner $\mathbf{n}_{2}, \mathbf{n}_{3}, \ldots$ As proven in Proposition 3 (File S1), we can explicitly compute each $\mathbb{E}_{\mathbf{n}_{i}}\left(\mathbf{X}_{T_{i}}^{b}\right)$, which, combined with the previously mentioned computation of $\mathbb{E}_{\mathbf{n}_{i}}\left(T_{i}\right)$ for any initial condition $\mathbf{n}_{i}$, enables us to obtain the desired sequence $\mathbb{E}_{\mathbf{n}_{0}}\left(T_{0}\right), \mathbb{E}_{\mathbf{n}_{1}}\left(T_{1}\right), \ldots$ Figure 3 illustrates this result and provides a visualization of the mutational-meltdown effect in a single beneficial lineage for different values of $U$, $s_{\mathrm{b}}$, and $s_{\mathrm{d}}$.

\section{Theory 2: Beneficial lineages in an evolving population}

Until now, we have examined the process of lineage contamination in isolation; that is, the accumulation of deleterious mutations occurring after the production of a beneficial mutation. In addition, we have assumed that we know the selective advantage of the focal mutation and that deleterious mutations were all of the same effect.

In real populations, however: (1) deleterious mutations can occur both after and before the appearance of a beneficial mutation, and (2) the selective effects of mutations will not be constant, known values. Deleterious mutations that appear

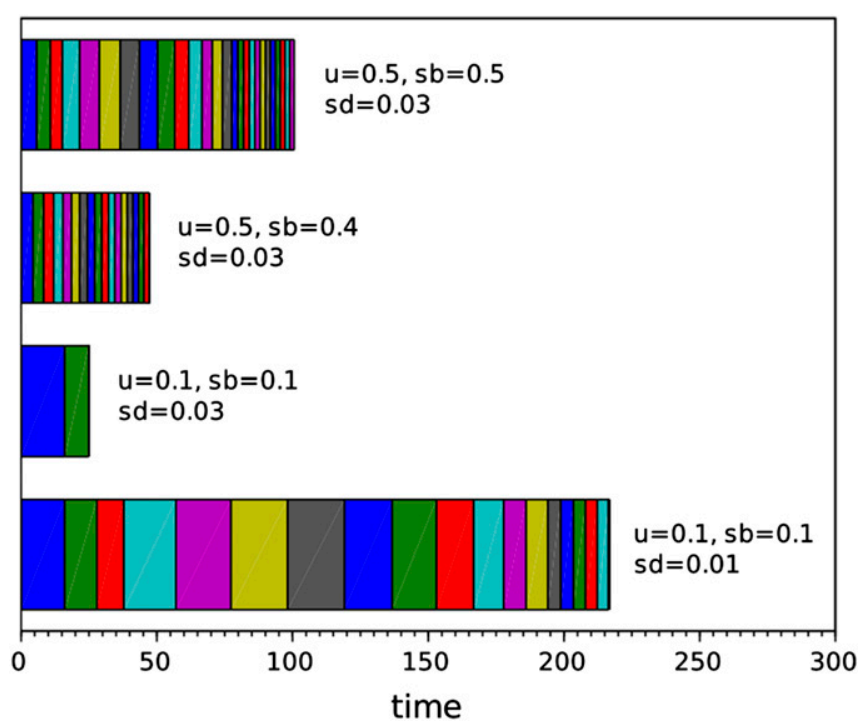

Figure 3 Mutational meltdown of beneficial lineages. Sequence $\mathbb{E}_{\mathbf{n}_{0}}\left(T_{0}\right), \mathbb{E}_{\mathbf{n}_{1}}\left(T_{1}\right), \ldots$ of the mean extinction times of the least-loaded classes, for one single beneficial lineage.

beforehand create a deleterious background upon which the beneficial mutation arises; selection against this deleterious background is background selection (Charlesworth et al. 1993; Stephan 2010). Here, we model the growth and fate of beneficial mutations of varying selective advantages arising in a population already contaminated with deleterious mutations. We further make a standard assumption of population genetics models here; namely, that the population is of constant size.

Angled-bracket notation in this section indicates average over all possible trajectories, or "states," (ensemble average) of a beneficial lineage emerging in a heterogeneous (evolving) population. (The absence of angled brackets indicates that the focal beneficial lineage arises in an otherwise homogeneous population, as in the previous section.)

\section{Deleterious background}

We define random variable $I$ to denote the number of deleterious mutations present in a randomly-chosen genome. The fitness of this genome is thus $\prod_{k=1}^{I}\left[1-S_{\mathrm{d}}(k)\right]$, where each $S_{\mathrm{d}}(k)$ is randomly drawn from the distribution of deleterious mutational effects. We will assume: (1) that fitness effects of deleterious mutations are independent enough that, conditioned on $I=i, \mathbb{E}\left\{\prod_{k=1}^{i}\left[1-S_{\mathrm{d}}(k)\right]\right\} \approx\left(1-\bar{s}_{\mathrm{d}}\right)^{i}$; and (2) that the fitness of a genome with $i$ deleterious mutations is approximately equal to this expectation. Mean fitness of the population is thus $\mathbb{E}_{I}\left[\left(1-\bar{s}_{\mathrm{d}}\right)^{I}\right]$.

Given that a randomly chosen genome carries $I=i$ deleterious mutations, replication of this genome in subsequent generations will form a branching process whose mean offspring number is initially equal to its own fitness divided by the mean fitness of the population, or: $\left(1-\bar{s}_{\mathrm{d}}\right)^{i} / \mathbb{E}_{I}\left[\left(1-\bar{s}_{\mathrm{d}}\right)^{I}\right]$ This fact is necessitated by the assumption of constant population size. Under this scheme, classical theory (Haigh 1978; 



Figure 4 Ensemble survival probability predictions compared to singlelineage simulations. Solid curves plot the bounds delineated by Equation 9. Points each plot the fraction of 30,000 individual-based stochastic simulations in which the focal beneficial mutation survived. Lineage survival was defined as either achieving a size of 5000 or lasting for 5000 generations. Solid points plot simulation results for which no further beneficial mutation occurred $(c=0)$; Open $\bigcirc$ plots simulations that allowed further beneficial mutations to occur at rate $c=0.001$ times the deleterious rate $U$. Different values of mean effect of deleterious mutations, $\bar{S}_{\mathrm{d}}$, were used, indicated by the different colors. Dashed curves plot the lower bound predicted by Equation 8 in the two cases for which $\bar{s}_{\mathrm{d}}>\bar{s}_{\mathrm{b}}$. Beneficial mutations have mean selective advantage $\bar{s}_{\mathrm{b}}=0.05$. (A) Plots the case of binary fission $(K=4)$; (B) plots the case of Poissondistributed offspring $(K=2)$.

Johnson 1999b) predicts random variable $I$ to be asymptotically Poisson with parameter $\theta=U / \bar{s}_{\mathrm{d}}$. Our simulations show that this asymptotic result provides a surprisingly good approximation even when adaptive substitutions are occurring with considerable frequency. The validity of this asymptotic approximation may break down, however, when populations are small and/or mutation rates are high. To include the possibility of such conditions, our numerical solutions employ the more encompassing distribution derived by Gessler (1995) (rederived in File S1), which is also a function of $\theta$. To retain tractability, however, analytical results employ the Poisson asymptote. In general, we let $\mathcal{P}_{\theta}(i)$ denote the probability that a randomly-chosen genome carries $i$ deleterious mutations: $I \sim \mathcal{P}_{\theta}(i)$. And in either case, we have $\mathbb{E}_{I}\left[\left(1-\bar{s}_{\mathrm{d}}\right)^{I}\right]=e^{-U}$.

\section{Fitness and growth rate of a beneficial lineage}

The fitness advantage of a beneficial mutation in the present section is allowed to vary and is thus denoted here by the random variable $S_{\mathrm{b}}$, with mean $\bar{s}_{\mathrm{b}}=\mathbb{E}\left(S_{\mathrm{b}}\right)$. When a beneficial mutation appears, the relative fitness of the resulting genotype is initially $\left(1+S_{\mathrm{b}}\right) \prod_{k=1}^{I}\left[1-S_{\mathrm{d}}(k)\right]$. Given $S_{\mathrm{b}}=s_{\mathrm{b}}$ and $I=i$, and employing the expectation for the deleterious background, the initial fitness of the resulting genotype is given by the function:

$$
\bar{w}_{i}=\left(1+s_{\mathrm{b}}\right)\left(1-\bar{s}_{\mathrm{d}}\right)^{i} .
$$

Adhering to the assumption of constant population size, the lineage founded by this beneficial mutation will form a branching process whose mean offspring number (growth rate) is initially $\bar{w}_{i} / \mathbb{E}_{I}\left[\left(1-\bar{s}_{\mathrm{d}}\right)^{I}\right]=e^{U} \bar{w}_{i}$.

\section{Survival probability}

In the context of an evolving population, ensemble survival probability is computed from the general expression:

$$
\left\langle p_{\mathrm{svl}}\right\rangle=\sum_{i \in \mathrm{N}} \mathcal{P}_{\theta}(i) \int p_{\mathrm{svl}}\left(i, s_{\mathrm{b}}\right) g\left(s_{\mathrm{b}}\right) d s_{\mathrm{b}}
$$

where $g\left(s_{\mathrm{b}}\right)$ is the probability density of selective effects, $S_{\mathrm{b}}$, of beneficial mutations and $p_{\mathrm{svl}}\left(i, s_{\mathrm{b}}\right)$ is the conditional survival probability, given that the beneficial mutation in question has selective advantage $S_{\mathrm{b}}=s_{\mathrm{b}}$ and arises in an individual carrying $i$ deleterious mutations. Integration limits and the normalizing constant for $g\left(s_{\mathrm{b}}\right)$ are determined by the support of $p_{\text {svl }}\left(i, s_{\mathrm{b}}\right)$ and are thus model dependent; for small $\bar{s}_{\mathrm{b}}$ and $g\left(s_{\mathrm{b}}\right)$ not heavy tailed, however, integration over $\mathbb{R}^{+}$ provides a very good approximation.

The conditional survival probability $p_{\text {svl }}\left(i, s_{\mathrm{b}}\right)$ is computed in the same way as $p_{\text {svl }}$ was computed in the context of lineage contamination in isolation (previous section), but whereas initial growth rate in the previous section was $1+s_{\mathrm{b}}$, it is now $e^{U} \bar{w}_{i}$. Because the values of $i$ and $s_{\mathrm{b}}$ will determine the number of equations required to compute $p_{\text {svl }}\left(i, s_{\mathrm{b}}\right)$, summation over $i$ and integration over $s_{\mathrm{b}}$ will require a numerical approach. In File S1, we employ a Monte Carlo approach that simultaneously performs both sum and integral.

\section{Bounds}

When neither $\bar{s}_{\mathrm{d}}$ nor $\bar{s}_{\mathrm{b}}$ are large, we have found a compact approximation that quite accurately puts bounds on survival probabilities. If a beneficial mutation arises on a genetic background carrying $i$ deleterious mutations and has selective advantage $s_{\mathrm{b}}$, our multitype branching process model puts bounds on survival probability as follows:

$$
p_{\mathrm{svl}}\left(i, s_{\mathrm{b}}\right)=K c_{1} \bar{w}_{i}^{-1}\left(1-c_{1} \bar{w}_{i}^{-1}\right)^{+},
$$

where upper and lower bounds are determined by the value of $c_{1}$ chosen; $c_{1}=e^{-U}$ for $\bar{s}_{\mathrm{d}} \rightarrow 0$ (upper bound), and $c_{1}=1$ for $\bar{s}_{\mathrm{d}} \geq 1-1 /\left(1+\bar{s}_{\mathrm{b}}\right)$ (lower bound) or for $\bar{s}_{\mathrm{d}} \geq \bar{s}_{\mathrm{b}}$ when $\bar{s}_{\mathrm{b}}$ is less than $\sim 0.2$. The superscript " + " denotes $\max \{\cdot, 0\}$.

We define a new function, $g^{*}(y \mid i)=g\left(y+\ln c_{1}+i \bar{s}_{\mathrm{d}}\right)$, and its Laplace transform $\mathscr{G}(\Phi \mid i)=\mathcal{L}\left\{g^{*}(y \mid i)\right\}$ (assuming it exists). Bounds on ensemble survival probability of beneficial 
mutations are then well approximated by the general expression:

$$
\left\langle p_{\text {svl }}\right\rangle=K \mathbb{E}_{I}[\mathscr{G}(1 \mid I)-\mathscr{G}(2 \mid I)],
$$

where $I \sim \mathcal{P}_{\theta}(i)$, and again where upper and lower bounds are determined by the value of $c_{1}$ chosen.

There are statistical arguments deriving from extremevalue theory (Smid and Stam 1975; Gillespie 1991; Rozen et al. 2002; Orr 2003) suggesting that selective advantages of beneficial mutations, owing to their definition as a quotient of higher-order statistics minus one, are exponentially distributed, i.e., $g\left(s_{\mathrm{b}}\right)=\left(1 / \bar{s}_{\mathrm{b}}\right) e^{-s_{\mathrm{b}} / \bar{s}_{\mathrm{b}}}$ (cf Rokyta et al. 2008). In this case, we find:

$$
\left\langle p_{\text {svl }}\right\rangle= \begin{cases}K \hat{s}_{\mathrm{b}} e^{-U / \bar{s}_{\mathrm{d}}\left(1-e^{-\bar{s}_{\mathrm{d}} / \bar{s}_{\mathrm{b}}}\right),}, & \bar{s}_{\mathrm{d}} \gtrsim \bar{s}_{\mathrm{b}} \\ K \hat{s}_{\mathrm{b}}, & \bar{s}_{\mathrm{d}} \rightarrow 0,\end{cases}
$$

where $\hat{s}_{\mathrm{b}}=\bar{s}_{\mathrm{b}} /\left[\left(1+\bar{s}_{\mathrm{b}}\right)\left(1+2 \bar{s}_{\mathrm{b}}\right)\right]$. (See File S1 for full derivation.) The foregoing expression indicates that the smallest value of $\left\langle p_{\text {svl }}\right\rangle$ is achieved when $\bar{s}_{\mathrm{d}} \approx \bar{s}_{\mathrm{b}}$, so that survival probability is bounded as:

$$
K \hat{s}_{\mathrm{b}} e^{-\varepsilon U / \bar{s}_{\mathrm{b}}} \lesssim\left\langle p_{\mathrm{svl}}\right\rangle \lesssim K \hat{s}_{\mathrm{b}},
$$

where $\varepsilon=1-e^{-1} \approx 0.63$. Remarkably: (1) the foregoing bounds on survival probability are independent of $\bar{s}_{\mathrm{d}}$, and (2) these bounds accurately contain survival probabilities from simulations under a wide variety of different conditions (File S1, Figure 4, and Figure 5). From (8) and Figure 6, we can see that the upper bound in (9) is approximated in the extremes of $\bar{s}_{\mathrm{d}}$, because: (1) when $\bar{s}_{\mathrm{d}}$ is small the upper bound is approximated; and (2) when $\bar{s}_{\mathrm{d}} \gtrsim \bar{s}_{\mathrm{b}}$ the lower bound is obtained, but as $\bar{s}_{\mathrm{d}}$ becomes increasingly larger than $\bar{s}_{\mathrm{b}}$, $\left\langle p_{\text {svl }}\right\rangle$ moves away from the lower bound and back toward the upper bound. This is because, as deleterious mutations have increasingly large effect, they are removed more efficiently from the population, leaving a large fraction of the population free of deleterious mutations. On the other hand, for intermediate values of $\bar{s}_{\mathrm{d}}$, or more precisely for $\bar{s}_{\mathrm{d}}$ in the vicinity of $\bar{s}_{\mathrm{b}}$, the lower bound is approximated.

\section{Selective advantages of surviving beneficial mutations}

Because of lineage contamination, beneficial mutations of small effect have a very small chance of survival; beneficial mutations that do survive, therefore, will tend to be of larger selective advantage. Following logic similar to that of the previous subsection, we derive the ensemble survival probability for a beneficial mutation of given selective advantage $s_{\mathrm{b}}$. Normalizing this probability, we derive the expected selective advantage of surviving beneficial mutations (File S1). Letting $S_{\mathrm{b}}^{*}$ denote the selective advantage of a surviving beneficial mutation, we find:

$$
\mathbb{E}\left(S_{\mathrm{b}}^{*}\right)=a+b U
$$

where $a=\hat{s}_{\mathrm{b}}\left(2+3 \bar{s}_{\mathrm{b}}\right)$ and $b=e^{-\bar{s}_{\mathrm{d}} / \bar{s}_{\mathrm{b}}}$ for $\bar{s}_{\mathrm{d}} \gtrsim \bar{s}_{\mathrm{b}}$ or $b=0$ for $\bar{s}_{\mathrm{d}} \rightarrow 0$. We note that, while selective advantages of surviving
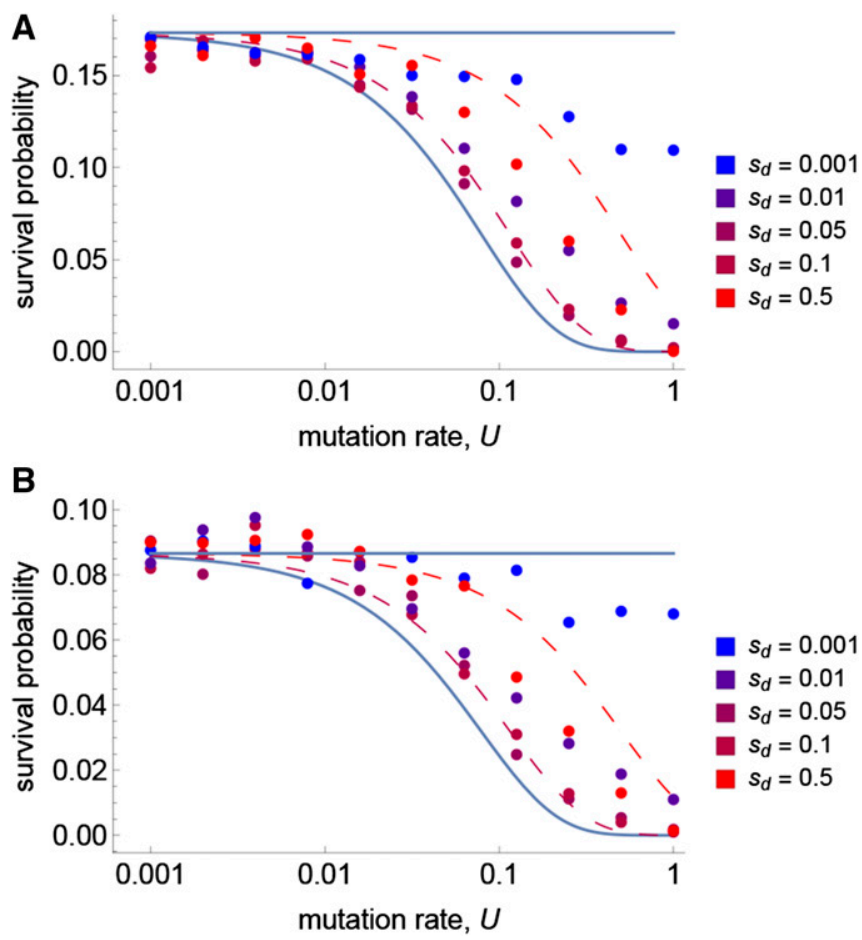

Figure 5 Ensemble survival probability predictions compared to fixation probabilities in whole-population simulations. Solid curves plot the bounds delineated by Equation 9. Points each plot the fraction of 5000 simulated Wright-Fisher populations in which a focal beneficial mutation achieved fixation. Each simulated population started out at the classical mutation-selection balance predicted by Haigh (1978) and ran for 100 generations before a single beneficial mutation was ascribed to a randomly chosen individual and tracked in subsequent generations. No other beneficial mutations appeared in the population (mutation was deleterious only). The 100-generation lead time was implemented to allow for any deviations from classical mutation-selection balance to acrue-deviations such as those described by Gessler (1995). Population size was 10,000, and the focal beneficial mutation had a fitness advantage drawn at random from an exponential distribution with mean $\bar{s}_{b}=0.05$. Different values of mean effect of deleterious mutations, $\bar{s}_{d}$, were used, as indicated by color scheme. Dashed curves plot the lower bound predicted by Equation 8 in the two cases for which $\bar{s}_{d}>\bar{s}_{b}$. (A) Plots the case of binary fission $(K=4)$; (B) plots the case of Poissondistributed offspring $(K=2)$.

beneficial mutations can increase with $U$, their recruitment rate decreases with $U$ : at high mutation rates, surviving beneficial mutations will tend to be of large effect but they will be rare.

\section{Mutation rate that maximizes production of surviving beneficial mutations}

The recruitment rate of beneficial mutations increases with genomic mutation rate, but because of lineage contamination, the survival probability of beneficial mutations decreases with genomic mutation rate. Therefore, there must exist a genomic mutation rate that maximizes the rate of production of surviving beneficial mutations. Setting $\partial_{U} U\left\langle p_{\text {svl }}\right\rangle=0$ and solving for $U$, we find this maximum production rate occurs at mutation rate $\hat{U}$, bounded as: 

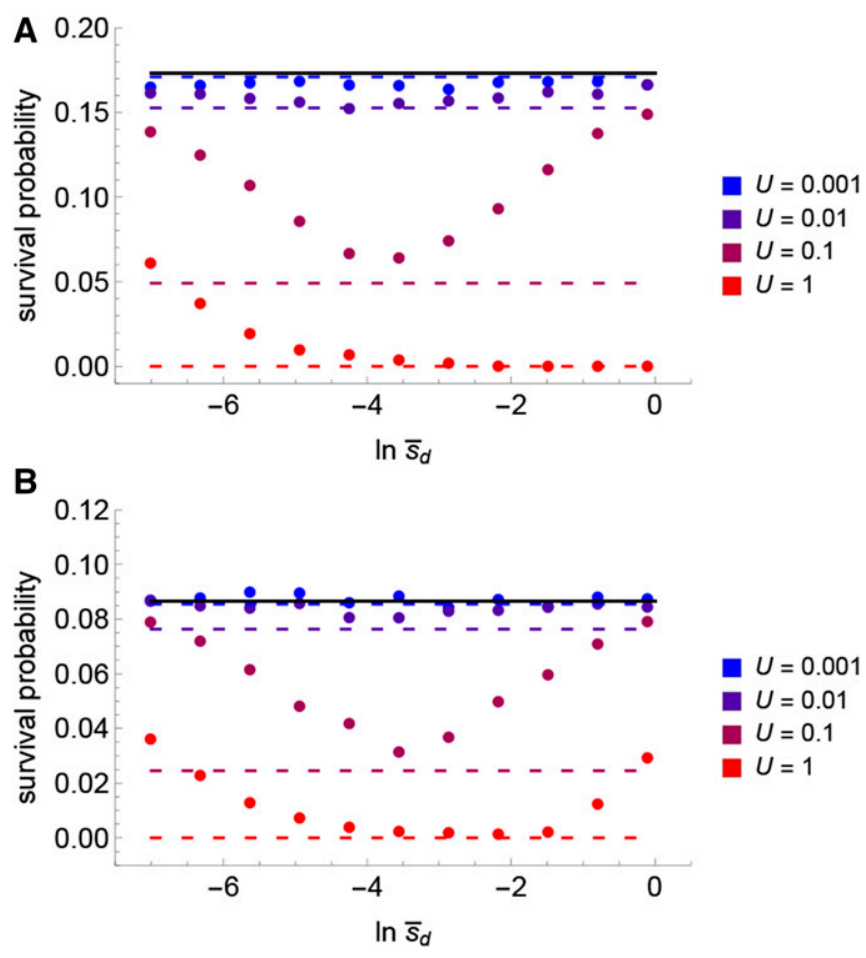

Figure 6 Ensemble survival probability. Predicted bounds compared to single-lineage simulations, as a function of mean selective disadvantage of deleterious mutations, $\bar{s}_{\mathrm{d}}$. Top black solid line plots the upper bound $K \hat{s}_{b}$; bottom dashed lines plot lower bounds $K \hat{s}_{b} e^{-\varepsilon U / \bar{s}_{b}}$ for the different values of $U$ indicated by the legend (Equation 8). Each point plots survival probabilities computed from 30,000 single-lineage simulations. Beneficial mutations have mean selective advantage $\bar{s}_{b}=0.05$. (A) Plots the case of binary fission $(K=4)$; (B) plots the case of Poisson-distributed offspring $(K=2)$.

$$
\hat{U}= \begin{cases}\bar{s}_{\mathrm{d}} /\left(1-e^{-\bar{s}_{\mathrm{d}} / \bar{s}_{\mathrm{b}}}\right), & \bar{s}_{\mathrm{d}} \geq \bar{s}_{\mathrm{b}} \\ \infty, & \bar{s}_{\mathrm{d}} \rightarrow 0 .\end{cases}
$$

Figure 7 compares the foregoing predictions to simulation results and shows them to be quite accurate. The smallest value of $\hat{U}$ is achieved when $\bar{s}_{\mathrm{d}}=\bar{s}_{\mathrm{b}}$, resulting in the bound:

$$
\hat{U} \geq \frac{1}{\varepsilon} \bar{s}_{\mathrm{b}},
$$

again displaying a curious independence of $\bar{s}_{\mathrm{d}}$. By some definitions (see Discussion), this lower bound on the optimal mutation rate is already above the "error threshold." This observation, however, must not be overinterpreted. It has been shown that, under certain idealized conditions, optimal mutation rate can closely approximate the more relevant Nash equilibrium mutation rate in large asexual populations (Ishii et al. 1989), but under more realistic conditions a Nash equilibrium does not exist (André and Godelle 2005; Gerrish et al. 2007).

\section{Theory 3: Effects of excessive mutation}

The lineage-contamination effect we describe will increase with increasing deleterious mutation rate (Figure 8). When

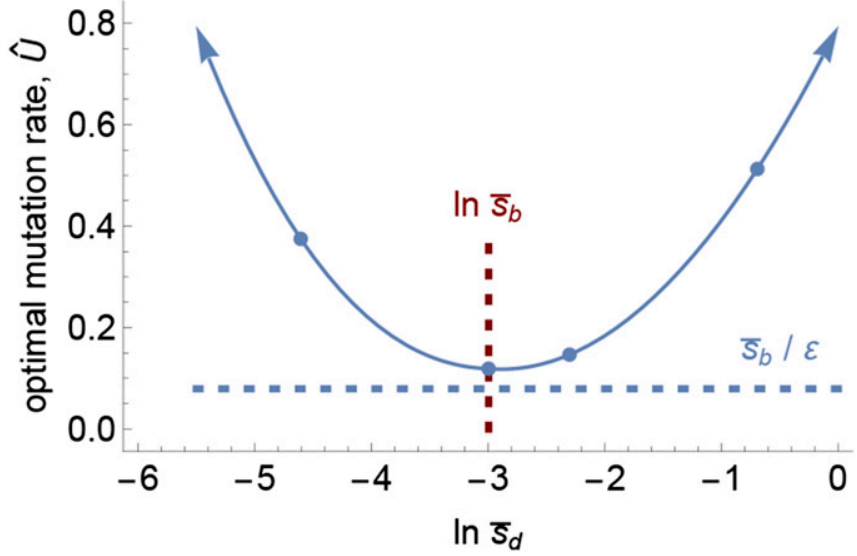

Figure 7 Optimal mutation rate, $\hat{U}$, as a function of $\ln \bar{S}_{d}$, compared with the theoretical minimum for this optimal mutation rate, $\bar{s}_{b} / \varepsilon$ (dashed blue line). Optimal mutation rate is defined as the deleterious mutation rate that maximizes the production rate of surviving beneficial mutations (Equation 11). The deleterious mutation rate is assumed to be proportional to beneficial mutation rate, but the constant of proportionality is irrelevant ( $\hat{U}$ is independent of this constant). Beneficial mutations have mean selective advantage $\bar{s}_{b}=0.05$, indicated by the red dashed vertical line. Each point represents an optimum computed as the $U$ which satisfies $\partial_{U} U p_{\text {svl }}(U)=0$. Here, $p_{\text {svl }}(U)$ is generated by interpolation of values of $p_{\text {svl }}$ each computed from 30,000 stochastic, individual-based simulations, for 20 different values of $U$.

the mutation rate is high enough, and when deleterious mutations are primarily of intermediate fitness effect (i.e., not at the extreme of small or large effect), lineage contamination can cause the within-population mutational meltdown of many newly arising beneficial lineages. This, in turn, can cause a reduction in competition among beneficial mutations, i.e., it can reduce clonal interference. At even higher mutation rates, this effect can suppress most or all newly arising beneficial lineages, resulting in the partial or complete cessation of adaptive evolution.

\section{Clonal interference threshold}

Evolutionary dynamics may be naturally partitioned into different regimes, depending on the recruitment rate of beneficial mutations. At very low rates of recruitment of beneficial mutations, adaptive evolution proceeds through isolated selective sweeps-a regime that has been dubbed the periodic selection regime (Sniegowski and Gerrish 2010). As the recruitment rate of beneficial mutations increases, a point is reached at which two or more alternative beneficial mutations may coexist and compete for fixation (the clonal-interference regime; Gerrish and Lenski 1998). As recruitment rate of beneficials continues to increase, it may become likely that competition occurs not among single beneficial mutations but among genotypes carrying multiple beneficial mutations (the "multiple-mutations clonal interference" regime; Desai and Fisher 2007; Desai et al. 2007).

What much of this previous work failed to account for ( $c f$. Orr 2000; Bachtrog and Gordo 2004) was the fact that, as 


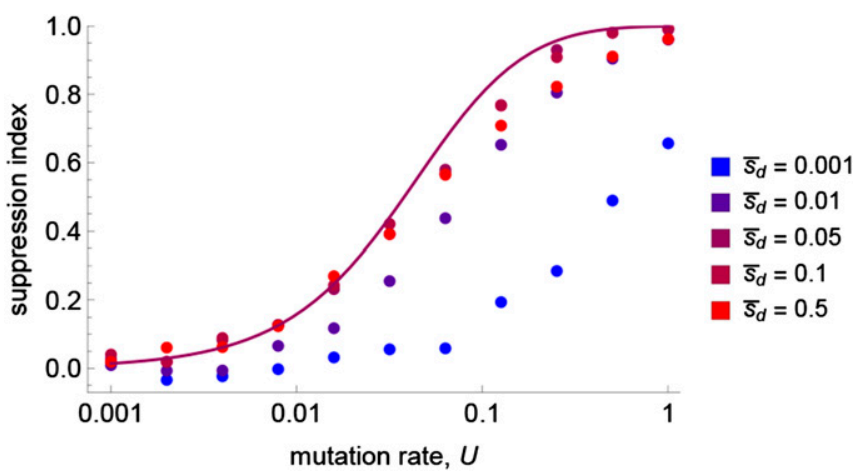

Figure 8 The contribution of lineage contamination to suppression of survival probabilities, defined as the complement of the factor (multiplier) of survival probability owed to lineage contamination. Concretely, survival probability may be written as $\left\langle p_{\text {svl }}\right\rangle=K \hat{s}_{\mathrm{b}} p_{\mathrm{LC}} p_{\mathrm{BS}}$, where $p_{\mathrm{LC}}$ and $p_{\mathrm{BS}}$ are the factors by which lineage contamination and background selection, respectively, suppress survival probability. These factors are smaller when suppression of survival probability is greater. Plotted here, therefore, is an index of such suppression due to lineage contamination, $1-p_{\mathrm{LC}}$, as a function of $U$. Points each plot the fraction of 30,000 single-lineage simulations. Solid curve plots predicted contribution for the case $\bar{s}_{\mathrm{d}}=\bar{s}_{\mathrm{b}}$, where $\bar{s}_{\mathrm{b}}=0.05$. Different values of mean effect of deleterious mutations, $\bar{s}_{d}$, were used, as indicated by color scheme. Offspring distribution was Poisson $(K=2)$.

beneficial recruitment rate increases via an increase in overall genomic mutation rate, the rate of deleterious mutation should increase in parallel. The findings we have presented so far suggest an intriguing implication of this parallel increase: whereas beneficial recruitment rate increases linearly with genomic mutation rate, survival probability of beneficial mutations decreases exponentially with genomic mutation rate. This fact suggests that, at high genomic mutation rates, the effects of lineage contamination can overwhelm the increased production of beneficial mutations, such that the effective recruitment rate of beneficials (i.e., the rate of production of surviving beneficial mutations) can decrease as mutation rate increases further. As mutation rate increases, therefore, adaptive evolution may eventually revert to a regime in which it proceeds only through isolated selective sweeps; put differently, the population may revert from one of the clonal-interference regimes back to the periodic-selection regime at high mutation rates.

As delineated in Sniegowski and Gerrish (2010), the clonal-interference regime is entered when a second, alternative beneficial mutation is likely to be produced on the ancestral background before the first, or focal, beneficial mutation becomes fixed. Mathematically, this transition occurs at the mutation rate $U$ that satisfies:

$$
n_{c}\langle U\rangle\left\langle p_{\text {svl }}\right\rangle=1,
$$

where $n_{c}(U)=N c U \ln \left[N \mathbb{E}\left(S_{\mathrm{b}}^{*}\right) / 2\right] / \mathbb{E}\left(S_{\mathrm{b}}^{*}\right)=1$ (File S1), where $c$ is thus the ratio of beneficial to deleterious rates. In previous work, analyzing the transition that occurs at low mutation rates, classical expressions for $\left\langle p_{\text {svl }}\right\rangle$ were used $\left(2 \bar{s}_{\mathrm{b}}\right.$, or some variant thereof) that are independent of $U$. Here, we have shown that, at high mutation rates and intermediate $\bar{s}_{\mathrm{d}},\left\langle p_{\text {svl }}\right\rangle$ can depend strongly on $U$, and Equation 13 can thus have more than one solution. We define the clonal-interference threshold to be the second of these solutions: the critical mutation rate above which adaptive evolution reverts from a clonal-interference regime back to a periodic-selection regime (Figure 9).

\section{Fixation threshold}

The critical selective advantage below which a beneficial mutation does not survive (almost surely when lineage contamination is considered in isolation, and in expectation when background selection is also accounted for) increases approximately linearly with mutation rate. In contrast, the fittest mutation produced by a population has a selective advantage that increases at most linearly with the log of the mutation rate. This necessarily implies that, as mutation rate increases, eventually a point will be reached at which even the selective advantage of the fittest beneficial mutation will not be sufficient to overcome the effects of lineage contamination. This point defines the "fixation threshold," and its existence follows from the fact that the critical selective advantage required and the maximum selective advantage produced by a population have qualitatively different relationships with mutation rate.

The fixation threshold is exceeded when no beneficial mutation produced by a population has a selective advantage strong enough to survive the effects of lineage contamination. Concretely, in a given interval of time $\tau$, we suppose a population with mutation rate $U$ produces a total of $n_{f}(U)$ beneficial mutations; then, the fixation threshold is defined as the mutation rate that prevents fixation of all of these beneficial mutations with specified probability $p_{c}$. It is thus the mutation rate $U$ which satisfies:

$$
\left\langle p_{\text {ext }}\right\rangle^{n_{f}(U)}=p_{\mathrm{c}},
$$

where $n_{f}(U)=N U c \tau$ and $\tau$ denotes the relevant time period; for example, to compute the mutation rate at which, over a time period of 5000 generations, all fixations will be suppressed with probability $95 \%$, we set $\tau=5000$ and $p_{\mathrm{c}}=0.95$ (Figure 10).

\section{Data availability}

The authors state that all data necessary for confirming the conclusions presented in the article are represented fully within the article.

\section{Discussion}

Evolutionary interactions between linked deleterious and beneficial mutations have received increasing attention in recent years. It is now well accepted, for example, that background selection caused by the continual rain of deleterious mutations into regions of low recombination decreases the 

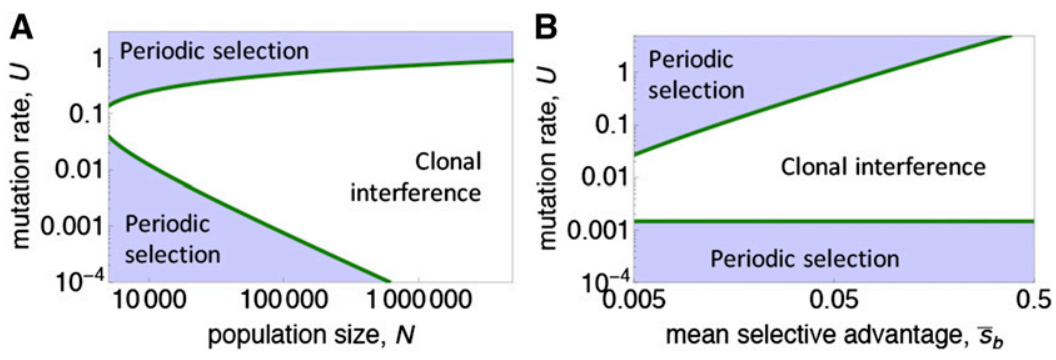

Figure 9 Clonal interference threshold. Mutation rates that delineate boundaries between clonal-interference and periodic-selection regimes, as computed from Equation 13. The greater of the two solutions (top green solid curves) represents the transition, as high mutation rate increases, from a clonal-interference regime back to a periodic selection regime (the clonal-interference threshold); the lesser of the two solutions (bottom green solid curves) represents the first transition, as low mutation rate increases, from a periodic selection regime into the clonal-interference regime. (A) Parameters are: $\bar{s}_{b}=0.05$ and $\bar{s}_{d}=0.06$. (B) Parameters are: $N=500,000$ and $\bar{s}_{d}=1.1 \times \bar{s}_{b}$. fixation probability of beneficial mutations (Charlesworth et al. 1993; Peck 1994) and decreases nucleotide diversity (Birky and Walsh 1988; Kim and Stephan 2000; Keightley and Otto 2006; Stephan 2010); moreover, theoretical and empirical studies have shown that selective sweeps of beneficial mutations can cause the fixation of linked deleterious mutations (Birky and Walsh 1988; Bachtrog and Gordo 2004; Hartfield et al. 2010; McDonald et al. 2011; Good and Desai 2014). To date, work in this area has been focused on populations with relatively low genomic mutation rates. In such populations, the key consideration in analyzing the interaction between beneficial and deleterious mutations is the number of deleterious mutations already present in the linked genomic background on which a new beneficial mutation arises. In the current article, we have focused, in contrast, on populations in which genomic mutation rates may be very high: we have examined the possibility that the genomic background on which a beneficial mutation arises can become progressively and disproportionately contaminated with newly arising deleterious mutations even as the beneficial mutation spreads into the larger population. We have found that this effect can predominate as mutation rate increases (Figure 8). Our work is motivated in part by numerous studies indicating that adapting asexual populations tend to evolve high mutation rates through genetic hitchhiking (Sniegowski et al. 1997, 2000; Johnson 1999a; Elena and Sanjuán 2005; M'Gonigle et al. 2009; Gentile et al. 2011; Raynes et al. 2011; Söderberg and Berg 2011), and by the substantial literature that has been devoted to the question of when the genomic mutation rate will be sufficiently high to cause population extinction (Eigen 1971, 2000, 2002; Eigen and Schuster 1977; Biebricher and Eigen 2005; Bull et al. 2007; Gerrish et al. 2007, 2013; Bull and Wilke 2008; Springman et al. 2009; Gerrish and Sniegowski 2012).


\section{Multiple beneficial mutations}

Our multitype branching process model assumes that beneficial mutations occur infrequently enough that acquiring a second beneficial mutation in linkage with the focal beneficial mutation is improbable in the time required for the focal mutation to either survive or go extinct. In reality, it might be the case that multiple beneficial mutations arise on the same background and sweep to fixation, collectively overcoming the lineage contamination effect.

To assess the strength of our assumption, we studied the effects of allowing additional beneficial mutations to arise at different rates within the lineage founded by the focal beneficial mutation. To this end we varied the parameter $c$, introduced above and defined as: $c=\mu / U$, or the ratio of numbers of potential beneficial to deleterious mutations. We assessed the effects of doing so in both an extension of our analytical model and in simulations.

To assess the effects of additional within-lineage beneficial mutations on lineage contamination in isolation, we extended our multitype branching process model so as to allow a beneficial lineage to acquire a second beneficial mutation with the same selective advantage $s_{\mathrm{b}}$ as the first. This additional beneficial mutation could be acquired during reproduction with probability $1-e^{-c U}$. Figure S9 in File S1 compares, for different values of $c$, survival probabilities of a single beneficial lineage in an otherwise homogeneous population (lineage contamination only) as a function of the deleterious mutation rate. It is apparent from this figure that, for reasonable values of $c$, there is minimal quantitative difference in survival probabilities and only a slight increase in the apparent threshold. This indicates that our assumption of no additional within-lineage beneficial mutations is a weak assumption. Mathematically, there is a qualitative difference in that, for $c>0$, the critical deleterious mutation rate above

Figure 10 Fixation threshold. Green curves plot fixation threshold defined as the deleterious mutation rate above which no fixations are predicted to occur over an interval of $\tau=1000$ generations, despite a parallel increase in beneficial mutation rate. These curves were generated by solving Equation 14. (A) Parameters are: $\bar{s}_{b}=0.05$ and $\bar{s}_{d}=0.06$. (B) Parameters are: $N=500,000$ and $\bar{s}_{\mathrm{d}}=1.1 \times \bar{s}_{\mathrm{b}}$. 
which a beneficial lineage becomes extinct almost surely (the "hard" threshold) is twice what it is without the additional beneficial mutation [i.e., $2 \ln \left(1+s_{\mathrm{b}}\right)$ ]. Practically, this is of little consequence, however, because the survival probabilities are typically minuscule for mutation rates in the region between the hard threshold for which no additional beneficial mutation is allowed $\left[\ln \left(1+s_{\mathrm{b}}\right)\right]$, and the hard threshold for which one additional beneficial mutation is allowed $\left[2 \ln \left(1+s_{\mathrm{b}}\right)\right]$. And, while not shown here, survival probabilities become even smaller in regions between higher thresholds that allow more beneficial mutations.

To assess the effects of additional within-lineage beneficial mutations arising in evolving populations, where both background selection and lineage contamination are operating, we performed simulations in which there was technically no limit on the number of additional beneficial mutations. Figure 4 plots survival probabilities computed from simulations, for the cases $c=0$, and 0.001 , and would seem to indicate, again, that our original assumption of no additional withinlineage beneficial mutation is a weak assumption: survival probabilities were essentially unaffected by the incorporation of additional beneficial mutations, except for very small values of $\bar{s}_{\mathrm{d}}$.

\section{Bounds independent of $\bar{s}_{\mathrm{d}}$}

The critical mutation rate above which lineage contamination in isolation ensures extinction of a beneficial mutation, derived in the first section (Equation 2), depends only on the selective advantage of the focal beneficial mutation; it does not depend on the selective disadvantages of deleterious mutations. When we incorporate background selection, most of the solution bounds we derive are also independent of the selective disadvantages of deleterious mutations. These results stand in contrast to some previously published results that have focused primarily on the effects of background selection. Particularly striking is the qualitative contrast between our results, which find a curious lack of dependence on $\bar{s}_{\mathrm{d}}$ in bounds we derive, and results of background-selection studies that instead find dependence on both $\bar{s}_{\mathrm{d}}$ and $s_{\mathrm{b}}$ (Birky and Walsh 1988; Charlesworth et al. 1993; Orr 2000; Charlesworth 2011) or even a curious lack of dependence on $s_{\mathrm{b}}$ (Orr 2000). For example, our approach to deriving the mutation rate that optimizes adaptation rate results in the expression $\hat{U} \geq \bar{s}_{\mathrm{b}} / \varepsilon$. Orr (2000), on the other hand, finds that $\hat{U} \approx \bar{s}_{\mathrm{d}}$. The single factor that accounts for the qualitative discrepancy between our results and these backgroundselection results is lineage contamination: when only background selection is accounted for, $\hat{U} \approx \bar{s}_{\mathrm{d}}$, yet when lineage contamination is also accounted for, $\hat{U} \geq \bar{s}_{\mathrm{b}} / \varepsilon$. The qualitative difference in dependencies of these results highlights the impact of lineage contamination.

\section{Lineage contamination in nature}

The effects of lineage contamination only become significant under linkage and relatively high mutation rates. While we have focused exclusively on the case of asexuality, lineage contamination should also operate in organisms that undergo some form of genetic exchange as well: the fitness of a newly arising beneficial mutation will be eroded at a faster rate than the same linkage region in the rest of the population-on principles similar to those studied here. The requirement of high mutation rates would seem to restrict the relevance of our findings to organisms like RNA viruses, although the evolution of high mutation rates has been predicted (Gerrish et al. 2007) and increasingly reported in natural (Matic et al. 1997) and laboratory (Cox and Gibson 1974; Sniegowski et al. 1997; Shaver et al. 2002; Wichman 2005; Pal et al. 2007; Chao and Cox 2008; Denver et al. 2009; Gentile et al. 2011) populations of RNA and clonal DNA organisms and in somatic (especially cancerous) cells. Indeed, the process we have analyzed has been implied in conjunction with background selection as a mechanism that can slow the evolution of tumors (Solé and Deisboeck 2004; McFarland et al. 2013).

\section{Lineage contamination, mutational meltdown, and lethal mutagenesis}

As alluded to in the presentation of our branching process model, lineage contamination may be thought of as withinpopulation mutational meltdown. If this meltdown is induced by treatment of a population with a mutagenic agent, then it may be thought of as within-population lethal mutagenesis. Put differently, our findings may be thought of as the population-genetic analogs of these processes. Indeed, one of the processes we model-Muller's ratchet in a growing beneficial lineage - is similar to previous models of Muller's ratchet in freely-growing populations (Fontanari et al. 2003; Bull et al. 2007; Bull and Wilke 2008). Our work differs from these previous studies, however, in that we model the fitness erosion of a growing lineage within the context of a larger population.

In a recent experiment, lethal mutagenesis failed to cause extinction in a laboratory population of the bacteriophage $\mathrm{T} 7$ (Springman et al. 2009) because the accumulation of deleterious mutations opened up new genetic pathways that could increase fitness, i.e., it increased the number of available beneficial mutations. The theory we present here may offer insight into what would be required to thwart the evolutionary rescue afforded by these newly available beneficial mutations. In particular, our fixation threshold might offer an appropriate quantitative guideline for the mutation rate required.

\section{Lineage contamination and the error threshold}

There is an intriguing relationship between our findings and predictions of error threshold models (Eigen 1971, 2002; Eigen and Schuster 1977; Nowak and Schuster 1989; Bonhoeffer and Stadler 1993; Bagnoli and Bezzi 1998; Biebricher and Eigen 2005). Generally and somewhat loosely speaking, an error threshold is a critical mutation rate, $U_{\mathrm{et}}$, above which all genotypes deterministically converge to the same equilibrium frequency, independent of their fitness (in the absence of mutational biases). The error-threshold model derives the 
ultimate deterministic fate of "fitness classes," whereas our lineage-contamination model follows the stochastic trajectory of individual lineages. Despite this fundamental difference in approach and questions addressed, there are some curious parallels in the critical mutation rates derived.

Single-peak model: The simplest model of the error threshold - the so-called "single-peak" model-assumes that there is a single fittest genotype of fitness $1+s_{\mathrm{b}}$ (the beneficial mutant) and all other (mutationally accessible) genotypes have fitness equal to one (Wiehe 1997; Tejero et al. 2011), i.e., the fitness landscape has a set of two possible fitness classes $\left\{1,1+s_{\mathrm{b}}\right\}$ This fitness landscape is obviously unrealistic; its original conjecture may have been based on the fact that many other such "phase-transition" phenomena are robust to severe model simplification. When the number of possible genotypes may be assumed to be infinite, the error threshold is $U_{\mathrm{et}}=\ln \left(1+s_{\mathrm{b}}\right)$ (Wiehe 1997). Curiously, while the assumed fitness landscapes are very different, this critical mutation rate is identical to the one we derive for lineage contamination in isolation (Equation 2).

Multiplicative model: The set of possible fitness classes on the "multiplicative" fitness landscape is $\left\{\left(1+s_{\mathrm{b}}\right)\left(1-s_{\mathrm{d}}\right)^{i} \quad \forall \quad i=0,1,2, \ldots, D\right\}$, where $D$ is the maximum number of deleterious mutations allowed. Curiously, for the case $D<+\infty$ and $s_{\mathrm{b}}=s_{\mathrm{d}}$, the error threshold is $U_{\text {et }}=s_{\mathrm{b}}$ [we note that for small $s_{\mathrm{b}}, U_{\mathrm{et}} \approx \ln \left(1+s_{\mathrm{b}}\right)$, in agreement with the single-peak model]; whereas when $D=+\infty$, there is no error threshold: $U_{\text {et }}=+\infty$ (Wiehe 1997). Oddly, our lineage-contamination model corresponds most closely to the case $D=+\infty$, for which there is no error threshold, but there is a lineage-contamination threshold.

\section{Concluding remarks}

Wittingly or not, the presence of lineage contamination has been implicit in many previous models of mutation-induced fitness erosion. To our knowledge, however, it has not previously been modeled in isolation, as a process separate from background selection. Our theoretical framework partitions these two processes and allows lineage contamination to be scrutinized separately from other processes. This new angle has provided new insights and results. We find, for example, that newly arising beneficial mutations can be driven extinct almost surely by lineage contamination; whereas background selection alone cannot ensure their extinction.

\section{Acknowledgments}

We thank Thomas Bataillon, Guillaume Martin, Alan Perelson, Nick Hengartner, Thomas Burr, and Eduarda Pimentel for helpful discussions; two anonymous referees; and Bill Gilson (University of New Mexico-Los Alamos) for vital computer support. S.P. received financial support from the "Soutien à la recherche des jeunes maîtres de conférences" program at the Université Paris-
Est Créteil. P.G. carried out much of this work in, and received financial support from, visiting faculty programs at Aarhus University, Denmark, and at the Institute of Evolutionary Sciences (ISEM) and the Mediterranean Center for Environment and Biodiversity (Labex/CeMEB) at the University of Montpellier, France. P.S. and P.G. received financial support from National Aeronautics and Space Administration grant NNA15BB04A.

\section{Literature Cited}

André, J., and B. Godelle, 2005 The evolution of mutation rate in finite asexual populations. Genetics 172: 611-626.

Bachtrog, D., and I. Gordo, 2004 Adaptive evolution of asexual populations under Muller's ratchet. Evolution 58: 1403-1413.

Bagnoli, F., and M. Bezzi, 1998 Eigen's error threshold and mutational meltdown in a quasispecies model. Int J Mod Phys 9: 999-1005.

Barton, N. H., 1995 Linkage and the limits to natural selection. Genetics 140: 821-841.

Barton, N. H., 2009 Why sex and recombination? Cold Spring Harb. Symp. Quant. Biol. 74: 187-195.

Biebricher, C. K., and M. Eigen, 2005 The error threshold. Virus Res. 107: 117-127.

Birky, C. W., and J. B. Walsh, 1988 Effects of linkage on rates of molecular evolution. Proc. Natl. Acad. Sci. USA 85: 6414-6418.

Bonhoeffer, S., and P. Stadler, 1993 Error thresholds on correlated fitness landscapes. J. Theor. Biol. 164: 359-372.

Bull, J. J., and C. O. Wilke, 2008 Lethal mutagenesis of bacteria. Genetics 180: 1061-1070.

Bull, J. J., R. Sanjuán, and C. O. Wilke, 2007 Theory of lethal mutagenesis for viruses. J. Virol. 81: 2930-2939.

Campos, P., 2004 Fixation of beneficial mutations in the presence of epistatic interactions. Bull. Math. Biol. 66: 473-486.

Campos, P. R. A., and L. M. Wahl, 2010 The adaptation rate of asexuals: deleterious mutations, clonal interference and population bottlenecks. Evolution 64: 1973-1983.

Chao, L., and E. Cox, 2008 Competition between high and low mutating strains of Escherichia coli. Evolution 37: 125-134.

Charlesworth, B., 2009 Fundamental concepts in genetics: effective population size and patterns of molecular evolution and variation. Nat. Rev. Genet. 10: 195-205.

Charlesworth, B., 2011 The Effects of deleterious mutations on evolution at linked sites. Genetics 190: 5-22.

Charlesworth, B., 2013 Background selection 20 years on: the Wilhelmine E. key 2012 invitational lecture. J. Hered. 104: 161-171.

Charlesworth, B., M. T. Morgan, and D. Charlesworth, 1993 The effect of deleterious mutations on neutral molecular variation. Genetics 134: 1289-1303.

Cox, E. C., and T. C. Gibson, 1974 Selection for high mutation rates in chemostats. Genetics 77: 169-184.

Denver, D. R., P. C. Dolan, L. J. Wilhelm, W. Sung, J. I. Lucas-Lledo et al., 2009 A genome-wide view of Caenorhabditis elegans base-substitution mutation processes. Proc. Natl. Acad. Sci. USA 106: 16310-16314.

Desai, M. M., and D. S. Fisher, 2007 Beneficial mutation selection balance and the effect of linkage on positive selection. Genetics 176: 1759-1798.

Desai, M., D. Fisher, and A. Murray, 2007 The speed of evolution and maintenance of variation in asexual populations. Curr. Biol. 17: 385-394.

Eigen, M., 1971 Selforganization of matter and the evolution of biological macromolecules. Naturwissenschaften 58: 465-523. 
Eigen, M., 2000 Natural selection: a phase transition? Biophys. Chem. 85: 101-123.

Eigen, M., 2002 Error catastrophe and antiviral strategy. Proc. Natl. Acad. Sci. USA 99: 13374-13376.

Eigen, M., and P. Schuster, 1977 The hypercycle. A principle of natural self-organization. Part A: emergence of the hypercycle. Naturwissenschaften 64: 541-565.

Elena, S. F., and R. Sanjuán, 2005 Adaptive value of high mutation rates of RNA viruses: separating causes from consequences. J. Virol. 79: 11555-11558.

Felsenstein, J., 1974 The evolutionary advantage of recombination. Genetics 78: 737-756.

Fontanari, J. F., A. Colato, and R. S. Howard, 2003 Mutation accumulation in growing asexual lineages. Phys. Rev. Lett. 91: 218101.

Gentile, C. F., S. Yu, S. Serrano, P. J. Gerrish, and P. D. Sniegowski, 2011 Competition between high- and higher-mutating strains of Escherichia coli. Biol. Lett. 7: 422-424.

Gerrish, P. J., and R. E. Lenski, 1998 The fate of competing beneficial mutations in an asexual population. Genetica 102-103: 127-144.

Gerrish, P. J., and P. D. Sniegowski, 2012 Real time forecasting of near-future evolution. J. R. Soc. Interface 9: 2268-2278.

Gerrish, P. J., A. Colato, A. S. Perelson, and P. D. Sniegowski, 2007 Complete genetic linkage can subvert natural selection. Proc.Natl. Acad. Sci. USA 104: 6266-6271.

Gerrish, P. J., A. Colato, and P. D. Sniegowski, 2013 Genomic mutation rates that neutralize adaptive evolution and natural selection. J. R. Soc. Interface 10: 20130329.

Gessler, D. D., 1995 The constraints of finite size in asexual populations and the rate of the ratchet. Genet. Res. 66: 241-253.

Gillespie, J. H., 1991 The Causes of Molecular Evolution, Oxford University Press, Oxford.

Good, B. H., and M. M. Desai, 2014 Deleterious passengers in adapting populations. Genetics 198: 1183-1208.

Gordo, I., and B. Charlesworth, 2001 Genetic linkage and molecular evolution. Curr. Biol. 11: R684-R686.

Goyal, S., D. J. Balick, E. R. Jerison, R. A. Neher, B. I. Shraiman et al., 2012 Dynamic mutation-selection balance as an evolutionary attractor. Genetics 191: 1309-1319.

Haigh, J., 1978 The accumulation of deleterious genes in a population-Muller's ratchet. Theor. Popul. Biol. 14: 251267.

Haldane, J., 1927 A mathematical theory of natural and artificial selection. Part V. Selection and mutation. Proc. Camb. Philos. Soc. 23: 838-844.

Hartfield, M., S. Otto, and P. D. Keightley, 2010 The role of advantageous mutations in enhancing the evolution of a recombination modifier. Genetics 184: 1153-1164.

Ishii, K., H. Matsuda, Y. Iwasa, and A. Sasaki, 1989 Evolutionarily stable mutation rate in a periodically changing environment. Genetics 121: 163-174.

Jiang, X., Z. Xu, J. Li, Y. Shi, W. Wu et al., 2011 The Influence of deleterious mutations on adaptation in asexual populations. PLoS One 6: e27757-e27758.

Johnson, T., 1999a Beneficial mutations, hitchhiking and the evolution of mutation rates in sexual populations. Genetics 151: $1621-1631$.

Johnson, T., 1999b The approach to mutation-selection balance in an infinite asexual population, and the evolution of mutation rates. Proc. Biol. Sci. 266: 2389-2397.

Johnson, T., and N. H. Barton, 2002 The effect of deleterious alleles on adaptation in asexual populations. Genetics 162: 395-411.

Kaiser, V. B., and B. Charlesworth, 2009 The effects of deleterious mutations on evolution in non-recombining genomes. Trends Genet. 25: 9-12.
Keightley, P. D., and S. P. Otto, 2006 Interference among deleterious mutations favours sex and recombination in finite populations. Nature 443: 89-92.

Kim, Y., and W. Stephan, 2000 Joint effects of genetic hitchhiking and background selection on neutral variation. Genetics 155: 1415-1427.

Matic, I., M. Radman, F. Taddei, B. Picard, C. Doit et al., 1997 Highly variable mutation rates in commensal and pathogenic E. coli. Science 277: 1833-1834.

McDonald, M., T. F. Cooper, H. Beaumont, and P. Rainey, 2011 The distribution of fitness effects of new beneficial mutations in Pseudomonas fluorescens. Biol. Lett. 7: 98-100.

McFarland, C. D., K. S. Korolev, G. V. Kryukov, S. R. Sunyaev, and L. A. Mirny, 2013 Impact of deleterious passenger mutations on cancer progression. Proc. Natl. Acad. Sci. USA 110: 2910-2915.

McFarland, C. D., L. A. Mirny, and K. S. Korolev, 2014 Tug-of-war between driver and passenger mutations in cancer and other adaptive processes. Proc. Natl. Acad. Sci. USA 111: 1513815143.

M'Gonigle, L. K., J. J. Shen, and S. P. Otto, 2009 Mutating away from your enemies: the evolution of mutation rate in a hostparasite system. Theor. Popul. Biol. 75: 301-311.

Muller, H., 1950 Our load of mutations. Am. J. Hum. Genet. 2: 111-176.

Muller, H., 1964 The relation of recombination to mutational advance. Mutat. Res. 1: 2-9.

Nowak, M., and P. Schuster, 1989 Error thresholds of replication in finite populations mutation frequencies and the onset of Muller's ratchet. J. Theor. Biol. 137: 375-395.

Orr, H. A., 2000 The rate of adaptation in asexuals. Genetics 155: 961-968.

Orr, H. A., 2003 The distribution of fitness effects among beneficial mutations. Genetics 163: 1519-1526.

Pal, C., M. Maciá, A. Oliver, I. Schachar, and A. Buckling, 2007 Coevolution with viruses drives the evolution of bacterial mutation rates. Nature 450: 1079-1081.

Peck, J. R., 1994 A ruby in the rubbish: beneficial mutations, deleterious mutations and the evolution of sex. Genetics 137: 597-606.

Poon, A., and S. P. Otto, 2000 Compensating for our load of mutations: freezing the meltdown of small populations. Evolution 54: 1467-1479.

Raynes, Y., M. R. Gazzara, and P. D. Sniegowski, 2011 Mutator dynamics in sexual and asexual experimental populations of yeast. BMC Evol. Biol. 11: 158.

Rokyta, D. R., C. J. Beisel, P. Joyce, M. T. Ferris, C. L. Burch et al., 2008 Beneficial fitness effects are not exponential for two viruses. J. Mol. Evol. 67: 368-376.

Rozen, D. E., J. A. G. M. de Visser, and P. J. Gerrish, 2002 Fitness effects of fixed beneficial mutations in microbial populations. Curr. Biol. 12: 1040-1045.

Shaver, A. C., P. G. Dombrowski, J. Y. Sweeney, T. Treis, R. M. Zappala et al., 2002 Fitness evolution and the rise of mutator alleles in experimental Escherichia coli populations. Genetics 162: $557-566$.

Silander, O. K., O. Tenaillon, and L. Chao, 2007 Understanding the evolutionary fate of finite populations: the dynamics of mutational effects. PLoS Biol. 5: e94.

Smid, B., and A. J. Stam, 1975 Convergence in distribution of quotients of order statistics. Stochastic Process. Appl. 3: 287292.

Sniegowski, P. D., and P. J. Gerrish, 2010 Beneficial mutations and the dynamics of adaptation in asexual populations. Philos. Trans. R. Soc. Lond. B Biol. Sci. 365: 1255-1263.

Sniegowski, P. D., P. J. Gerrish, and R. E. Lenski, 1997 Evolution of high mutation rates in experimental populations of Escherichia coli. Nature 387: 703-705. 
Sniegowski, P. D., P. J. Gerrish, T. Johnson, and A. Shaver, 2000 Evolution of mutation rates: separating causes from consequences. Bioessays 22: 1057-1066.

Söderberg, R. J., and O. G. Berg, 2011 Kick-starting the ratchet: the fate of mutators in an asexual population. Genetics 187: 1129-1137.

Solé, R. V., and T. S. Deisboeck, 2004 An error catastrophe in cancer? J. Theor. Biol. 228: 47-54.

Springman, R., T. Keller, I. J. Molineux, and J. J. Bull, 2009 Evolution at a high imposed mutation rate: adaptation obscures the load in phage T7. Genetics 184: 221-232.

Stephan, W., 2010 Genetic hitchhiking vs. background selection: the controversy and its implications. Philos. Trans. R. Soc. Lond. B Biol. Sci. 365: 1245-1253.
Sturtevant, A. H., 1937 Essays on evolution. I. On the effects of selection on mutation rate. Q. Rev. Biol. 12: 467-477.

Tejero, H., A. Marín, and F. Montero, 2011 The relationship between the error catastrophe, survival of the flattest, and natural selection. BMC Evol. Biol. 11: 2.

Wichman, H. A., 2005 Adaptive molecular evolution for 13,000 phage generations: a possible arms race. Genetics 170: 1931.

Wiehe, T., 1997 Model dependency of error thresholds: the role of fitness functions and contrasts between the finite and infinite sites models. Genet. Res. 69: 127-136.

Communicating editor: L. M. Wahl 